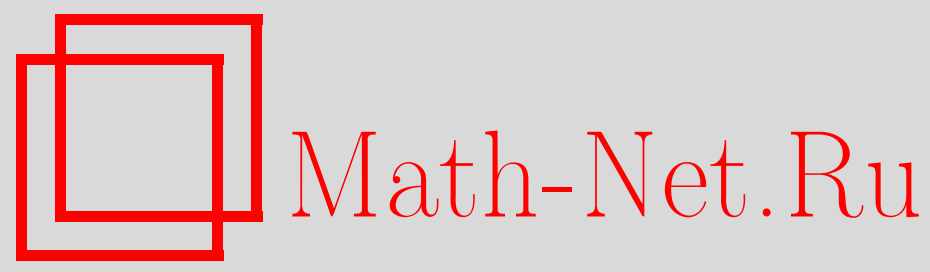

Е. А. Аусекле, Э. Ф. Оя, Теорема Питта для лоренцовых и орличевых пространств последовательностей, Матем. заметки, 1997, том 61, выпуск 1, 18-25

DOI: https://doi.org/10.4213/mzm1478

Использование Общероссийского математического портала Math-Net.Ru подразумевает, что вы прочитали и согласны с пользовательским соглашением http://www . mathnet.ru/rus/agreement

Параметры загрузки:

IP: 34.227 .88 .159

26 апреля 2023 г., 12:13:12

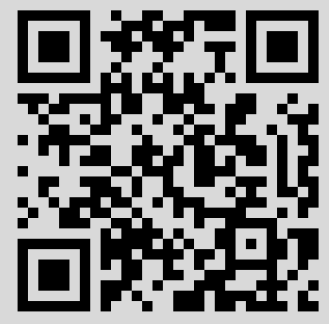




\title{
ТЕОРЕМА ПИТТА ДЛЯ ЛОРЕНЦОВЫХ И ОРЛИЧЕВЫХ ПРОСТРАНСТВ ПОСЛЕДОВАТЕЛЬНОСТЕЙ
}

\author{
Е.А. Аусекле, Э.Ф. Оя
}

Пусть $L(X, Y)$ - банахово пространство всех непрерывных линейных операторов из $X$ в $Y$, а $K(X, Y)$ - его подпространство компактных операторов. Устанавливаются варианты классической теоремы Питта (если $p>q$, то $\left.K\left(\ell_{p}, \ell_{q}\right)=L\left(\ell_{p}, \ell_{q}\right)\right)$ для подпространств лоренцовых и орличевых пространств последовательностей.

Библиограффия: 6 названий.

1. Пусть $L(X, Y)$ - банахово пространство всех непрерывных линейных операторов, действующих из банахова пространства $X$ в банахово пространство $Y$, а $K(X, Y)$ - его подпространство компактных операторов из $X$ в $Y$. Пусть $1 \leqslant p, q<\infty$.

Согласно классической теореме Питта $K\left(\ell_{p}, \ell_{q}\right)=L\left(\ell_{p}, \ell_{q}\right)$, если $p>q$ (см., например, $[1$, c. 76$])$. Здесь пространства $\ell_{p}$ и $\ell_{q}$ могут быть заменены их произвольными подпространствами (см., например, [2, с. 129]). С другой стороны, ясно, что $K\left(\ell_{p}, \ell_{q}\right) \neq$ $L\left(\ell_{p}, \ell_{q}\right)$, если $p \leqslant q$.

Одним из ближайших аналогов пространства $\ell_{p}$ считается лоренцово пространство последовательностей $d(v, p)$. Напомним его определение (см., например, [1], где можно найти все необходимые нам факты о лоренцовых пространствах последовательностей).

Пусть $v=\left(v_{k}\right)=\left(v_{k}\right)_{k=1}^{\infty}$ - такая невозрастающая последовательность положительных чисел, что $v_{1}=1, \lim v_{k}=0$ и

$$
\sum_{k=1}^{\infty} v_{k}=\infty
$$

Лоренцово пространство последовательностей $d(v, p)$ - это банахово пространство всех числовых последовательностей $x=\left(\xi_{k}\right)$, для которых конечна норма

$$
\|x\|=\sup _{\pi}\left(\sum_{k=1}^{\infty} v_{k}\left|\xi_{\pi(k)}\right|^{p}\right)^{1 / p}
$$

где супремум берется по всем перестановкам $\pi$ натуральных чисел $\mathbb{N}$.

Работа выполнена при финансовой поддержке Эстонского фонда науки, гранты № 762 и № 1381. 
Пространства $d(v, p)$ и $\ell_{p}$ не являются изоморфными. Тем не менее, они обладают многими одинаковьми свойствами. Например, любое бесконечномерное подпространство пространств $\ell_{p}$ или $d(v, p)$ содержит подпространство, изоморфное $\ell_{p}$.

Орличевы пространства последовательностей (см. определение в п. 5) являются обобщением пространств $\ell_{p}$. Для орличевых пространств последовательностей имеет место полный аналог теоремы Питта (см. [1, с. 149], а также теорему 3 ниже и замечание 2 к ней). Однако, аналог теоремы Питта для лоренцовых пространств последовательностей (как будет показано в п. 3) верен лиш в следуюшей форме.

ТЕОРема 1. Пусть $X$ и $Y$ - подпространства пространств $d(v, p)$ u $d(w, q)$ соответственно. Если $p>q u w \notin \ell_{p /(p-q)}$, mо $K(X, Y)=L(X, Y)$.

Имеет место также следующий уточняющий результат (которьй доказьвается в п. 4).

Теорема 2. Пусть $X=d(v, p) u Y=d(w, q)$. Ecлu $p>q u w \in \ell_{p /(p-q)}$ uлu же $p \leqslant q$, то $K(X, Y) \neq L(X, Y)$, причем $K(X, Y)$ не является дополняемым в $L(X, Y)$.

Несколько слов об обозначениях, принятых в настоящей работе. В работе рассматриваются банаховы пространства над одним и тем же полем $\mathbb{K}$ вещественных или комплексных чисел. Подпространства банаховых пространств предполагаются замкнутыми.

Для замкнутой линейной оболочки последовательности $\left(x_{k}\right)$ в $X$ будет использоваться обозначение $\left[x_{k}\right]$. Через $X^{*}$ обозначается, как обычно, сопряженное пространство пространства $X$.

И, наконец, пусть $1<p<\infty$. Для $p$ определяется сопряженный показатель $p^{\prime}$ так, что $1 / p+1 / p^{\prime}=1$.

2. Для доказательства теорем 1 и 3 (теорема 3 формулируется в п. 5) нам понадобятся следующие ниже понятие $\alpha$-доминируемости последовательностей и предложение, дающее довольно общее достаточное условие для равенства $K(X, Y)=L(X, Y)$ в случае подпространств $X$ и $Y$ банаховых пространств с базисами.

Пусть $\alpha=\left(a_{k}\right)$ - последовательность чисел и пусть $\left(x_{k}\right)$ и $\left(y_{k}\right)$ - последовательности в некоторых банаховьх пространствах. Будем говорить, что $\left(x_{k}\right) \alpha$-доминирует $\left(y_{k}\right)$, если существует такое число $C>0$, что при любых $n \in \mathbb{N}$ имеет место неравенство

$$
\left\|\sum_{k=1}^{n} a_{k} y_{k}\right\| \leqslant C\left\|\sum_{k=1}^{n} a_{k} x_{k}\right\| \text {. }
$$

В этом случае будем писать $\left(x_{k}\right)>_{\alpha}\left(y_{k}\right)$.

Пусть $E$ - банахово пространство с базисом $\left(e_{k}\right)$. Напомним, что блок-базисом базиса $\left(e_{k}\right)$ назьвается последовательность элементов

$$
u_{k}=\sum_{j=m_{k}+1}^{m_{k+1}} c_{j} e_{j} \neq 0
$$

где $0 \leqslant m_{1}<m_{2}<\cdots$ и $c_{j} \in \mathbb{K}$. Блок-базис $\left(u_{k}\right)$ является базисной последовательностью в $E$. Напомним еще, что последовательности $\left(x_{k}\right)$ и $\left(y_{k}\right)$ в некоторых банаховых пространствах назьваются әквивалентными, и пишут $\left(x_{k}\right) \sim\left(y_{k}\right)$, если

$$
\left\{\left(\lambda_{k}\right): \sum_{k=1}^{\infty} \lambda_{k} x_{k} \text { сходится }\right\}=\left\{\left(\lambda_{k}\right): \sum_{k=1}^{\infty} \lambda_{k} y_{k} \text { сходится }\right\} .
$$


Если $\left(x_{k}\right)$ и $\left(y_{k}\right)$ - базисные последовательности, то они эквивалентны тогда и только тогда, когда существует изоморфизм $A$ из $\left[x_{k}\right]$ на $\left[y_{k}\right]$ такой, что $A x_{k}=y_{k}, k \in \mathbb{N}$. Весь необходимьй нам материал о базисных последовательностях можно найти в [1]. В частности, нам понадобится следующий результат Бессаги-Пелчинского (см. [1, с. 7]). Каждая последовательность $\left(x_{k}\right)$ в банаховом пространстве с базисом $\left(e_{k}\right)$ такая, что $x_{k} \rightarrow 0$ слабо, но $\left\|x_{k}\right\| \not \nrightarrow 0$, имеет подпоследовательность $\left(x_{n_{k}}\right)$, эквивалентную некоторому нормированному блок-базису базиса $\left(e_{k}\right)$.

ПРЕДЛОЖЕНИЕ. Пусть $\alpha=\left(a_{k}\right)$ - последовательность чисел $и$ пусть $\left(\varepsilon_{k}\right) u$ $\left(\varphi_{k}\right)$ - такие последовательности в некоторых банаховых пространствах, что $\left(\varepsilon_{k}\right)$ не $\alpha$-доминирует $\left(\varphi_{k}\right)$. Пусть, далее, $E$ и $F$ - банаховы пространства $c$

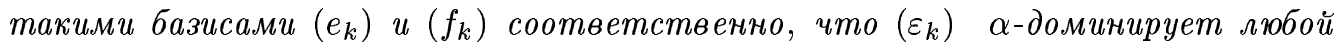
нормированный блок-базис $\left(u_{k}\right)$ базиса $\left(e_{k}\right)$, а любой нормированный блок-базис $\left(v_{k}\right)$ базиса $\left(f_{k}\right)$ имеет подпоследовательность $\left(v_{n_{k}}\right)>_{\alpha}\left(\varphi_{k}\right)$. Eсли $X$ и $Y-$ подпространства пространств $E$ и $F$ соответственно, причем $X^{*}$ сепарабельно, mo $K(X, Y)=L(X, Y)$.

ДоКАЗАтельство. Рассуждая от противного, допустим, что существует $T \in$ $L(X, Y) \backslash K(X, Y)$. Тогда имеется последовательность $\left(x_{k}\right) \subset X$ такая, что $x_{k} \rightarrow 0$ слабо, а $\left\|T x_{k}\right\| \geqslant C, k \in \mathbb{N}$, при некоторой константе $C>0$. Поэтому $T x_{k} \rightarrow 0$ слабо и $\left\|x_{k}\right\| \geqslant C /\|T\|$. Переходя к подпоследовательности последовательности $\left(x_{k}\right)$, а затем дважды в последовательности $\left(T x_{k}\right)$, можно считать, что $\left(x_{k}\right) \sim\left(u_{k}\right)$ и $\left(T x_{k}\right) \sim\left(v_{k}\right)>_{\alpha}\left(\varphi_{k}\right)$, где $\left(u_{k}\right)$ и $\left(v_{k}\right)$ - некоторые нормированные блок-базисы базисов $\left(e_{k}\right)$ и $\left(f_{k}\right)$ соответственно. Поскольку $\left(\varepsilon_{k}\right)>_{\alpha}\left(u_{k}\right) \sim\left(x_{k}\right)>_{\alpha}\left(T x_{k}\right)$, то $\left(\varepsilon_{k}\right)>_{\alpha}\left(\varphi_{k}\right)$, что невозможно.

Пусть $\left(\varepsilon_{k}\right)=\left(e_{k}\right)$ и $\left(\varphi_{k}\right)=\left(f_{k}\right)$ - естественные базисы в пространствах $E=\ell_{p}$ и $F=\ell_{q}$ соответственно, и пусть $\alpha=(1,1, \ldots)$. Ясно, что $\left(\varepsilon_{k}\right)$ не $\alpha$-доминирует $\left(\varphi_{k}\right)$, если $p>q$. Кроме того, любой нормированньй блок-базис естественного базиса в пространствах $\ell_{p}$ эквивалентен естественному базису (см., например, [1, с. 53]). Поэтому из предложения сразу вытекает теорема Питта для подпространств: ecли $p>q, m o$ $K(X, Y)=L(X, Y)$ при любых подпространствах $X \subset \ell_{p} u Y \subset \ell_{q}$.

3. ДоКАЗАТЕЛЬСТво теОРемы 1 . Пусть $\left(\varepsilon_{k}\right)$ и $\left(\varphi_{k}\right)$ - естественные базисы пространств $\ell_{p}$ и $d(w, q)$ соответственно. Заметим, что $p /(p-q)=(p / q)^{\prime}$. Поскольку $w=\left(w_{k}\right) \notin \ell_{(p / q)^{\prime}}=\left(\ell_{p / q}\right)^{*}$, то сушествует последовательность $\left(a_{k}\right) \in \ell_{p / q}, a_{1} \geqslant a_{2} \geqslant$ $\cdots>0$ такая, что

$$
\sum_{k=1}^{\infty} w_{k} a_{k}=\infty
$$

Пусть $\alpha=\left(a_{k}^{1 / q}\right)_{k=1}^{\infty}$. Допустим, что $\left(\varepsilon_{k}\right)>_{\alpha}\left(\varphi_{k}\right)$. Тогда при некоторой константе $C>0$ и произвольном $n \in \mathbb{N}$

$$
\begin{aligned}
\sum_{k=1}^{n} w_{k} a_{k} & =\left\|\sum_{k=1}^{n} a_{k}^{1 / q} \varphi_{k}\right\|_{d(w, q)}^{q} \leqslant C\left\|\sum_{k=1}^{n} a_{k}^{1 / q} \varepsilon_{k}\right\|_{\ell_{p}}^{q} \\
& =C\left(\sum_{k=1}^{n} a_{k}^{p / q}\right)^{q / p} \leqslant C\left\|\left(a_{k}\right)\right\|_{\ell_{p / q}}
\end{aligned}
$$


что невозможно. Значит, $\left(\varepsilon_{k}\right)$ не $\alpha$-доминирует $\left(\varphi_{k}\right)$.

Поскольку $d(v, p)$ рефлексивно и сепарабельно, то $d(v, p)^{*}$ сепарабельно. Поэтому и $X^{*}$ является сепарабельньм. Итак, в силу предложения остается показать, что там за пространство $E$ можно взять $d(v, p)$, а за пространство $F$ взять $d(w, q)$ вместе с их естественными базисами $\left(e_{k}\right)$ и $\left(f_{k}\right)=\left(\varphi_{k}\right)$ соответственно.

Хорошо известно (см., например, [1, с. 177]) и легко проверяется, что если $\left(u_{k}\right)$ - нормированньй блок-базис базиса $\left(e_{k}\right)$, то для любого набора чисел $\lambda_{1}, \ldots, \lambda_{n}$ вьполняется неравенство

$$
\left\|\sum_{k=1}^{n} \lambda_{k} u_{k}\right\|_{d(v, p)} \leqslant\left(\sum_{k=1}^{n}\left|\lambda_{k}\right|^{p}\right)^{1 / p}
$$

В частности, $\left(\varepsilon_{k}\right)>_{\alpha}\left(u_{k}\right)$.

Теперь рассмотрим произвольньй нормированный блок-базис $\left(u_{k}\right)$ базиса $\left(f_{k}\right)=$ $\left(\varphi_{k}\right) \subset d(w, q)$

$$
u_{k}=\sum_{j=m_{k}+1}^{m_{k+1}} c_{j} \varphi_{j}, \quad 0 \leqslant m_{1}<m_{2}<\cdots .
$$

Возможны два случая: $c_{j} \rightarrow 0$ и $c_{j} \not \rightarrow 0$.

Если $\lim c_{j}=0$, то $\left(u_{k}\right)$ содержит подпоследовательность $\left(u_{n_{k}}\right)$, которая эквивалентна естественному базису пространства $\ell_{q}$ (см. $[1$, с. 177]). Следовательно, существует $C>0$ такое, что для любого набора чисел $\lambda_{1}, \ldots, \lambda_{n}$

$$
C\left\|\sum_{k=1}^{n} \lambda_{k} u_{n_{k}}\right\|_{d(w, q)} \geqslant\left(\sum_{k=1}^{n}\left|\lambda_{k}\right|^{q}\right)^{1 / q} \geqslant\left\|\sum_{k=1}^{n} \lambda_{k} \varphi_{k}\right\|_{d(w, q)}
$$

(последнее неравенство очевидно), и, в частности, $\left(u_{n_{k}}\right)>_{\alpha}\left(\varphi_{k}\right)$.

Рассмотрим случай, когда $c_{j} \nrightarrow \nrightarrow 0$. Тогда $\left|c_{j_{n}}\right| \geqslant \varepsilon$ при некоторой последовательности $j_{1}<j_{2}<\cdots$ и некотором числе $\varepsilon>0$. Выделяя из $\left(u_{k}\right)$ подпоследовательность тех $\left(u_{k}\right)$, в представлении $(2)$ которых встречаются $c_{j_{n}}$, можем считать, что

$$
\forall k \in \mathbb{N} \exists n(k) \in\left\{m_{k}+1, \ldots, m_{k+1}\right\}: \quad\left|c_{n(k)}\right| \geqslant \varepsilon .
$$

Так как $\left(\varphi_{k}\right)$ - безусловньй базис с единичной безусловной базисной константой, то, в частности, при $b_{k}=a_{k}{ }^{1 / q}$ имеем

$$
\left\|\sum_{k=1}^{n} b_{k} u_{k}\right\|=\left\|\sum_{k=1}^{n} \sum_{j=m_{k}+1}^{m_{k+1}} c_{j} b_{k} \varphi_{j}\right\| \geqslant \frac{\varepsilon}{2}\left\|\sum_{k=1}^{n} b_{k} \varphi_{n(k)}\right\|=\frac{\varepsilon}{2}\left\|\sum_{k=1}^{n} b_{k} \varphi_{k}\right\|, \quad n \in \mathbb{N},
$$

т.е. $\left(u_{k}\right)>_{\alpha}\left(\varphi_{k}\right)$. Теорема 1 доказана.

ЗАМЕчАнИЕ. При доказательстве теоремы 1 мы использовали идеи, аналогичные примененным в доказательстве следствия 2 из [3]. 
4. ДОКАЗАТЕЛЬСТВо ТЕОРЕМЫ 2 использует факт (см. $[1$, с. 177$]$ ), что $d(v, p)$ (и даже любое его бесконечномерноеподпространство) содержит дополняемое подпространство, изоморфное $\ell_{p}$.

Пусть $X=d(v, p)$ и $Y=d(w, q)$, где $1 \leqslant p, q<\infty$. Рассмотрим случай, когда $p>q$ и $w \in \ell_{p /(p-q)}$. Покажем, что $K(X, Y) \neq L(X, Y)$. Так как существует непрерывный линейный проектор из $X$ на его подпространство, изоморфное $\ell_{p}$, то достаточно доказать, что $K\left(\ell_{p}, Y\right) \neq L\left(\ell_{p}, Y\right)$. Применяя неравенство Гёльдера и учитьвая, что $(p / q)^{\prime}=p /(p-q)$, для любого набора чисел $\lambda_{1}, \ldots, \lambda_{n}$ получим

$$
\begin{aligned}
\left(\sum_{k=1}^{n} w_{k}\left|\lambda_{k}\right|^{q}\right)^{1 / q} & \leqslant\left(\sum_{k=1}^{n} w_{k}^{(p / q)^{\prime}}\right)^{1 /\left((p / q)^{\prime} q\right)}\left(\sum_{k=1}^{n}\left(\left|\lambda_{k}\right|^{q}\right)^{p / q}\right)^{1 / p} \\
& \leqslant\left(\sum_{k=1}^{\infty} w_{k}^{p /(p-q)}\right)^{(p-q) /(p q)}\left(\sum_{k=1}^{n}\left|\lambda_{k}\right|^{p}\right)^{1 / p}
\end{aligned}
$$

Отсюда ясно, что $\ell_{p} \subset Y$, и формальньй единичньй оператор $T: \ell_{p} \rightarrow Y$ является непрерьвньм. Однако, $T$ не компактен, поскольку в случае естественного базиса $\left(e_{k}\right)$ пространства $\ell_{p}$ имеем, что $e_{k} \rightarrow 0$ слабо, но $\left\|T e_{k}\right\|=1 \not \rightarrow 0$.

То, что $K(X, Y) \neq L(X, Y)$, если $p \leqslant q$, следует из того, что $K\left(\ell_{p}, \ell_{q}\right) \neq L\left(\ell_{p}, \ell_{q}\right)$, а $X$ и $Y$ содержат дополняемые подпространства, изоморфные $\ell_{p}$ и $\ell_{q}$ соответственно.

Наконец, так как $K(X, Y) \neq L(X, Y)$ и $Y$ имеет безусловный базис, то $K(X, Y)$ является недополняемьм подпространством в $L(X, Y)$ (см. [4]). Теорема 2 доказана.

5. Теперь выведем из предложения (см. п. 2) аналог теоремы Питта для подпространств орличевых пространств последовательностей.

Напомним, что функиия Орлича $M$ - это заданная на $[0, \infty)$ непрерьвная неубьвающая выпуклая функция такая, что $M(0)=0, M(t)>0$ при $t>0$ и $\lim _{t \rightarrow \infty} M(t)=\infty$. Орличево пространство последовательностей $\ell_{M}$ определяется как совокупность всех числовых последовательностей $x=\left(\xi_{k}\right)$ таких, что найдется число $\rho=\rho(x)>0$, для которого

$$
\sum_{k=1}^{\infty} M\left(\frac{\left|\xi_{k}\right|}{\rho}\right)<\infty
$$

Пространство $\ell_{M}$ является банаховым относительно нормы

$$
\|x\|=\inf \left\{\rho>0: \sum_{k=1}^{\infty} M\left(\frac{\left|\xi_{k}\right|}{\rho}\right) \leqslant 1\right\} .
$$

Элементы $e_{1}=(1,0,0, \ldots), e_{2}=(0,1,0, \ldots), \ldots$ образуют в $\ell_{M}$ симметричную базисную последовательность, т.е. симметричньй базис для $h_{M}=\left[e_{k}\right] \subset \ell_{M}$. При этом $h_{M}=\ell_{M}$ тогда и только тогда, когда $M$ удовлетворяет $\Delta_{2}$-условию в нуле, т.е.

$$
\varlimsup_{t \rightarrow 0} \frac{M(2 t)}{M(t)}<\infty
$$


Пусть

$$
\begin{aligned}
& \alpha_{M}=\sup \left\{p>0: \sup \left\{\frac{M(\lambda t)}{M(\lambda) t^{p}}<\infty: 0<\lambda, t \leqslant 1\right\}\right\}, \\
& \beta_{M}=\inf \left\{q>0: \inf \left\{\frac{M(\lambda t)}{M(\lambda) t^{q}}>0: 0<\lambda, t \leqslant 1\right\}\right\} .
\end{aligned}
$$

Тогда $1 \leqslant \alpha_{M} \leqslant \beta_{M}$, причем $\beta_{M}<\infty$ тогда и только тогда, когда $M$ удовлетворяет $\Delta_{2}$-условию в нуле. Перечисленные, а также другие необходимые нам факты об орличевых пространствах последовательностей можно найти в [1].

Пространства $\ell_{M}$ являются обобшениями пространств $\ell_{p}$ : если взять $M(t)=t^{p}$, $p \geqslant 1$, то $\ell_{M}=\ell_{p}$. При этом, как легко показать, $\alpha_{M}=\beta_{M}=p$.

Tеорема 3. Пусть $X$ и $Y$ - подпространства орличевых пространств $h_{M} u$ $\ell_{N}$ соответственно. Если $\alpha_{M}>\beta_{N}$, то $K(X, Y)=L(X, Y)$.

ДокАЗАТЕЛЬСтво. Выберем числа $p$ и $q$ так, чтобы $\alpha_{M}>p>q \geqslant \beta_{N}$,

$$
M(\lambda t) \leqslant K M(\lambda) t^{p}, \quad 0<\lambda, t \leqslant 1,
$$

и

$$
k N(\lambda) t^{q} \leqslant N(\lambda t), \quad 0<\lambda, t \leqslant 1,
$$

при некоторых константах $k>0$ и $K>0$.

Пусть $\alpha=(1,1, \ldots)$ и пусть $\left(\varepsilon_{k}\right)$ и $\left(\varphi_{k}\right)$ - естественные базисы в пространствах $\ell_{p}$ и $\ell_{q}$ соответственно. Тогда $\left(\varepsilon_{k}\right)$ не $\alpha$-доминирует $\left(\varphi_{k}\right)$.

Так как $\alpha_{M}>1$, то сопряженная функция $M^{*}$ удовлетворяет $\Delta_{2}$-условию в нуле и, поэтому $h_{M}^{*}=\ell_{M^{*}}$ является сепарабельным (см. $[1$, с. 138,148$\left.]\right)$. Значит, и $X^{*}$ является сепарабельньм. Далее, поскольку $\beta_{N}<\infty$, то функция $N$ удовлетворяет $\Delta_{2}$-условию в нуле, так что $\ell_{N}=h_{N}$. Остается показать, что в предложении можно взять $E=h_{M}$ и $F=\ell_{N}$ вместе с их естественными базисами $\left(e_{k}\right)$ и $\left(f_{k}\right)$ соответственно.

Покажем, что для любого нормированного блок-базиса (1) базиса $\left(e_{k}\right)$ существует $C>0$ такое, что

$$
\nu_{n}=\left\|\sum_{k=1}^{n} u_{k}\right\|_{\ell_{M}} \leqslant C\left\|\sum_{k=1}^{n} \varepsilon_{k}\right\|_{\ell_{p}}=C n^{1 / p}, \quad n \in \mathbb{N},
$$

т.е. $\left(\varepsilon_{k}\right)>_{\alpha}\left(u_{k}\right)$.

Прежде всего заметим, что

$$
\begin{gathered}
\sum_{j=m_{k}+1}^{m_{k+1}} M\left(\left|c_{j}\right|\right)=1, \quad k \in \mathbb{N}, \\
\sum_{k=1}^{n} \sum_{j=m_{k}+1}^{m_{k+1}} M\left(\frac{\left|c_{j}\right|}{\nu_{n}}\right)=1, \quad n \in \mathbb{N},
\end{gathered}
$$


и $\nu_{1} \leqslant \nu_{2} \leqslant \cdots$. Из (6) ясно, что $\left|c_{j}\right| \leqslant \gamma, j \in \mathbb{N}$, при некоторой константе $\gamma \geqslant 1$. Для доказательства (5) нам достаточно рассматривать $\nu_{n} \geqslant \gamma$. Тогда согласно (3) при всех $j$

$$
M\left(\frac{\left|c_{j}\right|}{\nu_{n}}\right)=M\left(\frac{\left|c_{j}\right|}{\gamma} \frac{\gamma}{\nu_{n}}\right) \leqslant K M\left(\frac{\left|c_{j}\right|}{\gamma}\right) \gamma^{p} \nu_{n}^{-p} \leqslant K \gamma^{p} M\left(\left|c_{j}\right|\right) \nu_{n}^{-p}
$$

и поэтому согласно (7) и (6)

$$
1 \leqslant \sum_{k=1}^{n} K \gamma^{p} \nu_{n}^{-p} \sum_{j=m_{k}+1}^{m_{k+1}} M\left(\left|c_{j}\right|\right)=K \gamma^{p} n \nu_{n}^{-p},
$$

откуда вытекает (5).

Аналогично доказьвается (используя (4) и то, что $N$ удовлетворяет $\Delta_{2}$-условию в нуле), что $\left(v_{k}\right)>_{\alpha}\left(\varphi_{k}\right)$ при любом нормированном блок-базисе $\left(v_{k}\right)$ базиса $\left(f_{k}\right)$. Теорема 3 доказана.

ЗАмЕчАниЕ 1. В теореме 3 , вообще говоря, $h_{M}$ не может быть заменено пространством $\ell_{M}$. На самом деле, пусть, например, $M(t)=e^{t-1 / t}$ при $t>0$ и $M(0)=0$. Тогда $\alpha_{M}=\infty$. Так как $M$ не удовлетворяет $\Delta_{2}$-условию в нуле, то $\ell_{M}$ содержит подпространство $X$, изоморфное $\ell_{\infty}$. Пусть $P$ - непрерьвный линейньй проектор из $\ell_{M}$ на $X, T: X \rightarrow \ell_{\infty}$ - изоморфизм и $Q \in L\left(\ell_{\infty}, \ell_{2}\right)$ - сюръекция (см., например, [1, с. 105, c. 111]). Тогда $Q T P \in L\left(\ell_{M}, \ell_{2}\right) \backslash K\left(\ell_{M}, \ell_{2}\right)$.

ЗАмечание 2 . Частньй случай теоремы 3 , когда $M$ и $N$ удовлетворяют $\Delta_{2}$-условию в нуле, $X=\ell_{M}=h_{M}$ и $Y=\ell_{N}=h_{N}$, содержится в [1, с. 149]. Там же доказано, что если $M$ и $N$ удовлетворяют $\Delta_{2}$-условию в нуле и $\alpha_{M} \leqslant \beta_{N}$, то $K\left(\ell_{M}, \ell_{N}\right) \neq L\left(\ell_{M}, \ell_{N}\right)$.

6. Пусть $X$ и $Y$ - рефлексивные пространства. Согласно одной теореме Н. Калтона [5] равенство $K(X, Y)=L(X, Y)$ влечет за собой рефлексивность пространства операторов $L(X, Y)$. Поэтому из теорем 1 и 3 вытекают два нижеследующих результата.

СлЕДСТВИЕ 1. Пусть $X$ u $Y$ - подпространства пространств $d(v, p) u d(w, q)$ соответственно. Если $p>q>1 u w \in \ell_{p /(p-q)}$, то $L(X, Y)$ является рефлексивHblM.

СЛЕДСТВИЕ 2. Пусть $X$ и $Y$ - рефлексивные подпространства пространств $h_{M}$ и $\ell_{N}$ соответственно. Если $\alpha_{M}>\beta_{N}$, mо $L(X, Y)$ является рефлексивным.

Отметим, что $h_{M}$ (или $\left.\ell_{M}\right)$ является рефлексивным тогда и только тогда, когда функция $M$ и ее сопряженная функция $M^{*}$ удовлетворяют $\Delta_{2}$-условию в нуле. Это, в свою очередь, означает, что $\beta_{M}<\infty$ и $\alpha_{M}>1$.

Хорошо известно (см., например, [6, с. 247]), что из рефлексивности пространства $K(X, Y)$ следует равенство $K(X, Y)=L(X, Y)$, если одно из пространств $X$ или $Y$ удовлетворяет свойству аппроксимации. Поэтому, учитьвая теорему 2 и замечание $2 \mathrm{k}$ теореме 3 , имеем следующие результаты.

СлЕДСТвиЕ 3. Следующие утверждения равносильны:

1) $L(d(v, p), d(w, q))$ рефлексивно;

2) $K(d(v, p), d(w, q))$ рефлексивно;

3) $1<q<p u w \notin \ell_{p /(p-q)}$. 
СлЕДСТвиЕ 4. Следующие утверждения равносильны:

1) $L\left(\ell_{M}, \ell_{N}\right)$ рефлексивно;

2) $K\left(\ell_{M}, \ell_{N}\right)$ рефлексивно;

3) $1<\alpha_{N}, \beta_{N}<\alpha_{M}, \beta_{M}<\infty$.

Следствие 4, по-видимому, известно (однако, мы не можем дать точную ссылку). Для сравнения к следствиям 3 и 4 приведем хорошо известный частньй случай следствия 4 (см., например, [6, с. 248]), что $L\left(\ell_{p}, \ell_{q}\right)$ рефлексивно тогда и только тогда, когда $1<q<p<\infty$.

Авторы выражают благодарность Б. М. Макарову за внимание к работе.

Тартуский университет

Поступило

E-mail: jausekle@math.ut.ee, eveoja@math.ut.ee 28.08 .95

\section{СПИСОК ЦИТИРОВАННОЙ ЛИТЕРАТУРЫ}

[1] Lindenstrauss J., Tzafriri L. Classical Banach spaces. I. Sequence spaces. Berlin-Heidelberg-N. Y.: Springer, 1977.

[2] Lacey E. The isometric theory of classical Banach spaces. Berlin-Heidelberg-N. Y.: Springer, 1974.

[3] Oja E. On M-ideals of compact operators and Lorentz sequence spaces // Proc. Estonian Acad. Sci. Phys. Math. 1991. V. 40. №1. P. 31-36.

[4] Tong A. E., Wilken D. R. The uncomplemented subspace $K(E, F) / /$ Studia Math. 1971. V. 37. № 3. P. 227-236.

[5] Kalton N. J. Spaces of compact operators // Math. Ann. 1974. V. 208. № 4. P. 267-278.

[6] Diestel J., Uhl J. J., Jr. Vector measures. Providence: AMS, 1977. 